\title{
Usefulness of Ultrasonography in the Diagnosis of Peptic Ulcer Disease in Children
}

\author{
Eun Joo Lee, Yeoun Joo Lee, and Jae Hong Park \\ Department of Pediatrics, Pusan National University School of Medicine, Yangsan, Korea
}

\begin{abstract}
Purpose: This study was performed to assess the clinical usefulness of transabdominal ultrasonography (TUS) in detecting peptic ulcer disease (PUD) in children.

Methods: Twenty-four patients (19 boys, 5 girls; mean age, 10.6 \pm 4.5 years [range, 3.0-17.9 years]) who were admitted to the hospital for acute abdomen or gastrointestinal bleeding and diagnosed with PUD by endoscopy and who underwent TUS were included. Clinical data were retrospectively collected by reviewing patient medical records. Gastric ulcer (GU) was suspected when the gastric wall exceeded $8 \mathrm{~mm}$ in thickness and had lost its five-layer structure on TUS. Duodenal ulcer (DU) was suspected if the duodenal wall thickness exceeded $5 \mathrm{~mm}$.

Results: Sensitivity of TUS in diagnosing PUD was $66.7 \%$ for GU and $38.9 \%$ for DU. Mean age and body weight of the 11 patients suspected with PUD on TUS were $10.9 \pm 4.4$ years and $38.1 \pm 17.2 \mathrm{~kg}$, respectively. For 13 patients without suspected PUD, they were $12.1 \pm 4.1$ years and $39.6 \pm 17.0 \mathrm{~kg}$, respectively. There was a significant difference in height, weight, and body mass index between patients who were suspected to have PUD and those who were not suspected on TUS ( $p=0.014,0.008$, and 0.005 , respectively). A significant difference in the sensitivity of TUS in diagnosing PUD was found between patients under $30 \mathrm{~kg}$ and those over $30 \mathrm{~kg}(88.9 \%$ and $20.0 \%$, respectively; $p=0.003$ ).
\end{abstract}

Conclusion: TUS investigation of the stomach and duodenum is an efficient method for PUD detection in children with low body weight. TUS can be used in preliminary diagnostic work-up before further invasive tests.

Key Words: Ultrasonography, Stomach ulcer, Duodenal ulcer, Child

\section{INTRODUCTION}

Gastric and duodenal ulcers occur relatively infrequently in the pediatric population. Annual incidence rates of peptic ulcer disease (PUD) according to a systematic review of the literature published between 1997 and 2007 [1] were $0.10 \%$ to $0.19 \%$ for physician-diagnosed PUD. The rapidly declining prevalence of Helicobacter pylori infection and widespread use of potent anti-secretory drugs have sub-

Received : August 13, 2018, Revised : October 2, 2018, Accepted : October 9, 2018

Corresponding author: Jae Hong Park, Department of Pediatrics, Pusan National University Yangsan Hospital, 20 Geumo-ro, Mulgeum-eup, Yangsan 50612, Korea. Tel: +82-55-360-2180, Fax: +82-55-360-2181, E-mail: jhongpark@pusan.ac.kr

Copyright (c) 2019 by The Korean Society of Pediatric Gastroenterology, Hepatology and Nutrition

This is an open-access article distributed under the terms of the Creative Commons Attribution Non-Commercial License (http://creativecommons.org/licenses/by-nc/4.0/) which permits unrestricted non-commercial use, distribution, and reproduction in any medium, provided the original work is properly cited. 
stantially lowered the prevalence of PUD compared to that two decades ago [2]. However, there are only a few studies on the prevalence of PUD in children.

With respect to diagnostic tool of PUD, a single-contrast barium meal is not effective because of its low sensitivity and specificity. It is also difficult to perform in a timely manner. A double-contrast barium meal provides more accurate assessment of mucosal detail in the gastroduodenum than the single-contrast barium meal in radiographic study. However, most children under 6 years of age are not able to cooperate sufficiently for successful completion of an air-contrast examination [3]. This test is also less sensitive and specific than endoscopy.

Esophagogastroduodenoscopy is the standard modality for diagnosing PUD as it is very sensitive and highly specific in establishing the presence of mucosal ulceration both in the stomach and the duodenum [4]. While diagnostic upper gastrointestinal endoscopy can be performed at any time, especially if bleeding is the main symptom, routinely performing it in all patients with epigastric pain is neither time-efficient nor cost-efficient.

The presenting symptoms of PUD vary depending on the age of the patient. Hematemesis or melena is reported in up to half of patients with PUD [4]. Infants and younger children usually present with feeding difficulty, vomiting, crying episodes, hematemesis, or melena. The classic symptom of peptic ulceration (i.e., epigastric pain alleviated by the ingestion of food) is present only in a minority of children. Many pediatric patients present with poorly localized epigastric or periumbilical pain. As most of these patients do not have a peptic ulcer [4], clinical suspicion is the most important factor in the early diagnosis of PUD.

Transabdominal ultrasonography (TUS) is a valuable noninvasive tool for the diagnosis of intestinal lesions, including lesions in the stomach and duodenum. However, uncertainty remains concerning its accuracy as a primary imaging procedure in patients presenting with clinical suspicion of PUD. Although TUS is not the diagnostic test of choice for patients with PUD, many patients with PUD who present to the emergency department with unexplained abdominal pain or gastrointestinal (GI) bleeding undergo TUS as an initial diagnostic test. However, data regarding the usefulness of TUS in the early diagnosis of PUD in children are still lacking. Thus, the aim of this study was to determine the clinical usefulness of TUS in identifying patients with PUD in children.

\section{MATERIALS AND METHODS}

A total of 83 patients underwent both TUS and endoscopy because of acute abdomen with epigastric pain or upper GI bleeding between July 2010 and 2015 at Pusan National University Children's Hospital. Twenty-four patients ( 19 males, 5 females) were diagnosed with PUD by endoscopy. Clinical data such as sex, age, chief complaints, and the delay between symptom presentation and diagnosis of these 24 patients were collected retrospectively by reviewing patient medical records. This study was approved by the Institutional Review Board of Pusan National University Yangsan Hospital (approval number: 05-2018-153).

The sensitivity of TUS in diagnosing PUD, demographic and clinical characteristics of 11 patients suspected with peptic ulcers on TUS, demographic and clinical characteristics of 13 patients with a missed diagnosis of peptic ulcer on TUS, and the sensitivity of TUS in diagnosing PUD according to body weight were evaluated.

TUS was performed within 48 hours of hospital arrival in all patients. Examinations were conducted by a pediatric gastroenterologist and ultrasound specialist or radiologists. The thickness of the gastric and duodenal walls was examined by real-time ultrasound (Sequoia 512 US system; Acuson, Mountain View, CA, USA) using a high frequency probe (8-15 $\mathrm{MHz}$ linear transducers) and graded compression. Gastric ulcer (GU) was considered as a diagnosis when the gastric wall had a thickness that exceeded $8 \mathrm{~mm}$ and had lost its five-layer structure. Duodenal ulcer (DU) was diagnosed when the thickness of the duodenal wall exceeded $5 \mathrm{~mm}$. 
Comparisons of age, height, weight, and body mass index (BMI) between patients who were suspected to have PUD and those who were not suspected on TUS were analyzed using $t$-test while locations of lesions were analyzed using $\chi^{2}$ test. The sensitivity, specificity, and positive and negative predictive values of TUS in suspecting PUD according to body weight were evaluated using Fisher's exact test. The $p$-values less than 0.05 were considered statistically significant. Statistical analysis was performed using SPSS statistical software package for Windows ver. 21.0. (IBM Co., Armonk, NY, USA).

\section{RESULTS}

A total 24 patients ( 19 males, 5 females) were included in this study. The mean age of these patients was $10.6 \pm 4.5$ (range, 3.0-17.9) years. Ten (41.7\%) patients were over 13 years old (Table 1$)$.

Chief complaints were abdominal pain $(n=16$, $66.7 \%)$, hematemesis $(n=4,16.7 \%)$, vomiting $(n=3$, $12.5 \%)$, and melena $(n=1,4.2 \%)$. The delay between symptom presentation and diagnosis was 7.0 \pm 7.7 days. Fifteen $(62.5 \%)$ patients were diagnosed within one week after the onset of symptoms.

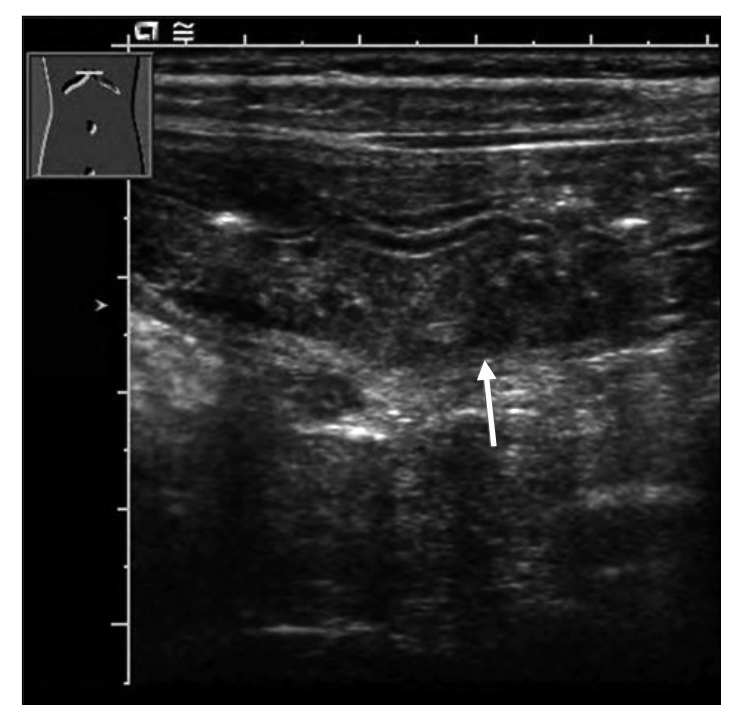

Fig. 1. Transverse gray scale sonogram through the gastric antrum demonstrating marked, circumferential gastric wall thickening with loss of the gastric five-layer structure (arrow).
In all patients diagnosed with PUD, the gastric antrum or duodenal bulb showed marked, diffuse, and circumferential wall thickening on TUS (Fig. 1 and 2 ). The 11 patients diagnosed with PUD had a mean age and body weight of $10.9 \pm 4.4$ years and $38.1 \pm 17.2 \mathrm{~kg}$, respectively. Four $(36.4 \%)$ patients had lesion on the gastric antrum, six $(54.5 \%)$ on the duodenal bulb, and one $(9.1 \%)$ on both gastric antrum and duodenal bulb. The 13 patients who were not diagnosed with PUD by TUS had a mean age and body weight of $12.1 \pm 4.1$ years and $39.6 \pm 17.0 \mathrm{~kg}$, respectively. The lesion was located at the gastric antrum in two (15.4\%) patients, at the duodenal bulb in nine $(69.2 \%)$, and on both the gastric antrum and the duodenal bulb in two $(15.4 \%)$. There were sig-

Table 1. Age and Sex Distribution of Patients

\begin{tabular}{cclc}
\hline Age $(\mathrm{y})$ & Male & Female & Total \\
\hline$\leq 3$ & $1(4.2)$ & $0(0.0)$ & $1(4.2)$ \\
$4-7$ & $7(29.2)$ & $1(4.2)$ & $8(33.3)$ \\
$8-12$ & $4(16.7)$ & $1(4.2)$ & $5(20.8)$ \\
$\geq 13$ & $7(29.2)$ & $3(12.5)$ & $10(41.7)$ \\
Total & $19(79.2)$ & $5(20.8)$ & $24(100.0)$ \\
\hline
\end{tabular}

Values are presented as number (\%). Mean age, $10.6 \pm 4.5$ (range, 3.0-17.9) years.

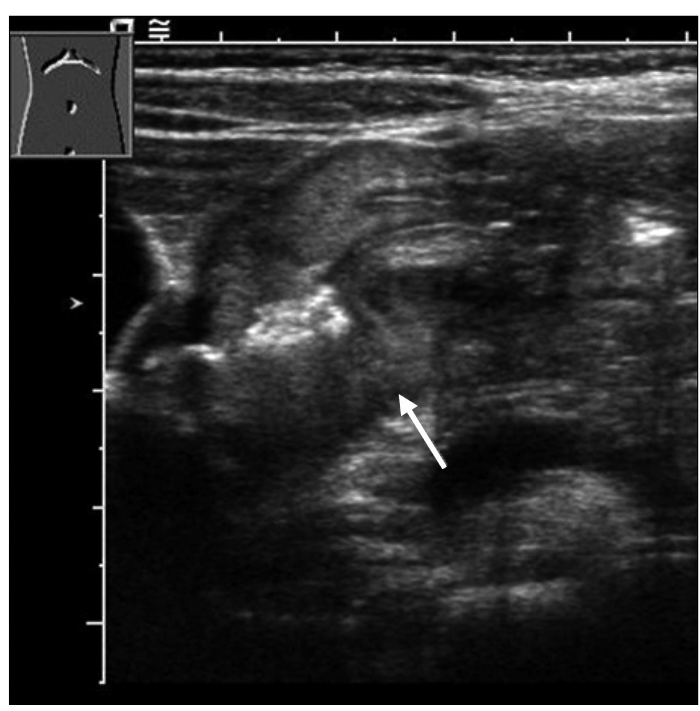

Fig. 2. Transverse gray scale sonogram through the duodenal bulb demonstrating hyperechoic center surrounded by a hypoechoic halo with various thicknesses (arrow). 
nificant differences in height, weight, and BMI between patients who were suspected to have PUD and those who were not suspected on TUS ( $p=0.014$, 0.008 , and 0.005 , respectively). However, there was no significant difference in age or the location of lesion between the two groups (Table 2).

The sensitivity of TUS in diagnosing PUD was $66.7 \%$ (4/6) for GU, $38.9 \%$ (7/18) for DU, and $45.8 \%$ (11/24) in total. We compared the sensitivity, specificity, and positive and negative predictive values of TUS for the diagnosis of PUD by dividing patients to those weighing less than $30 \mathrm{~kg}$ and those weighing more than $30 \mathrm{~kg}$. A significant difference in the sensitivity of TUS in diagnosing PUD was found between patients under $30 \mathrm{~kg}$ and those over $30 \mathrm{~kg}$ ( $88.9 \%$ vs. $20.0 \%, p=0.003)$. A significant difference in the negative predictive value of TUS was also found between the two groups $(96.3 \%$ vs. $70.7 \%$, $p=0.015)$. However, there was no significant difference in specificity or positive predictive value of TUS between the two groups $(p=0.747$ and 0.286 , respectively). There was no statistical difference in the sensitivity of TUS between the two groups according to the localization of the lesion $(p=0.479)$ (Table 3$)$.

\section{DISCUSSION}

Although PUD is rarely the etiology of abdominal pain in children, it must be excluded during the diagnosis process. To this end, TUS is now recognized as an important diagnostic tool in detecting abdominal disease as it can effectively be used to evaluate the stomach and duodenum [5,6]. Although it is not the first-line option in suspected PUD, it can be used in the diagnostic work-up before invasive tests. It may reduce diagnosis time in children. Early upper endoscopy is recommended in patients with PUD because it confirms the diagnosis and allows for targeted endoscopic treatment.

In the present study, the sensitivity of TUS in diagnosing PUD was $66.7 \%$ in GU and $38.9 \%$ in DU. Its sensitivity was much higher in patients under $30 \mathrm{~kg}$ $(88.9 \%)$ than that in patients over $30 \mathrm{~kg}(20.0 \%)$. To the best of our knowledge, this is the first study showing the diagnostic value according to body weight in children following their screening diagnostic TUS for suspected PUD.

The thickness of the normal GI tract varies considerably. The wall of the gastric antrum can measure up to $5 \mathrm{~mm}$ whereas that of the duodenum

Table 3. Sensitivity, Specificity, PPV, and NPV of TUS for Diagnosing PUD Lesion according to Body Weight

\begin{tabular}{ccccc}
\hline $\begin{array}{c}\text { Weight } \\
(\mathrm{kg})\end{array}$ & $\begin{array}{c}\text { Sensitivity } \\
(\%)\end{array}$ & $\begin{array}{c}\text { Specificity } \\
(\%)\end{array}$ & $\begin{array}{c}\text { PPV } \\
(\%)\end{array}$ & $\begin{array}{c}\text { NPV } \\
(\%)\end{array}$ \\
\hline$<30$ & $88.9^{*}$ & 96.3 & 88.9 & $96.3^{* *}$ \\
$\geq 30$ & 20.0 & 90.6 & 50.0 & 70.7 \\
\hline
\end{tabular}

PPV: positive predictive value, NPV: negative predictive value, TUS: transabdominal ultrasonography, PUD: peptic ulcer disease. ${ }^{*} p=0.003,{ }^{* *} p=0.015$.

Table 2. Comparisons of Demographic and Clinical Characteristics between Patients Suspected to Have PUD and Not Suspected on TUS

\begin{tabular}{lccc}
\hline \multicolumn{1}{c}{ Characteristic } & PUD positive $(\mathrm{n}=11)$ & PUD negative $(\mathrm{n}=13)$ & $p$-value \\
\hline Age $(\mathrm{y})$ & $10.6 \pm 4.5$ & $10.9 \pm 4.4$ & $143.2 \pm 23.8$ \\
Height $(\mathrm{cm})$ & $141.1 \pm 24.9$ & $39.6 \pm 17.0$ & $0.850^{*}$ \\
Body weight $(\mathrm{kg})$ & $38.1 \pm 17.2$ & $18.2 \pm 3.0$ & $0.080^{*}$ \\
Body mass index $\left(\mathrm{kg} / \mathrm{m}^{2}\right)$ & $17.9 \pm 3.0$ & $2(15.4)$ \\
Location of lesion $(\mathrm{n})$ & $4(36.4)$ & $9(69.2)$ \\
Gastric antrum & $6(54.5)$ & $2(15.4)$ \\
Duodenal bulb & $1(9.1)$ & $0.005^{*}$ \\
Both & &
\end{tabular}

Values are presented as mean \pm standard deviation or number $(\%)$.

PUD: peptic ulcer disease, TUS: transabdominal ultrasonography.

Analyzed by ${ }^{*} t$-test and ${ }^{\dagger} \chi^{2}$ test. 
and the small bowel usually measures 1 to $2 \mathrm{~mm}$ [7]. While inflammatory diseases such as gastritis or ulcers are usually not detected by ultrasonography, a circumscribed thickening of the gastric or duodenal wall with an echogenic center is occasionally seen in gastroduodenal ulcers $[8,9]$. In the present study, when the gastric wall had a thickness that exceeded $8 \mathrm{~mm}$ and had lost its five-layer structure, GU was suspected. When the thickness of the duodenal wall exceeded $5 \mathrm{~mm}$, DU was suspected.

Lorentzen et al. [10] have reported that gastric or duodenal wall thickening shown on TUS is a significant finding that indicates upper GI pathology in $86 \%$ of GU and $60 \%$ of DU cases. Computed tomography has demonstrated similar findings to TUS in the context of gastritis and PUD [11,12].

In the present study, the diagnosis rate of PUD using TUS was significantly different according to body weight. In the diagnosis of GI diseases, several studies have looked at the effect of body weight and age on the diagnostic yield of TUS. For example, Sulowski et al. [13] have shown that obesity is an independent predictor for a non-diagnostic screening TUS in suspected appendicitis. By using TUS to visualize the appendix in 126 children divided according to body weight (overweight, normal and underweight), another study [14] has shown that the appendix is not visible in $79 \%$ of overweight children. This percentage is significantly higher than that in the other weight groups. BMI also negatively influences the sensitivity of TUS in diagnosing appendicitis in children older than 14 years [15]. Yu et al. [16] have suggested that TUS could be useful in diagnosing acute appendicitis, especially in younger patients. In a study by Kotagal et al. [17], TUS was more likely to be non-diagnostic in overweight or older children in diagnosing acute appendicitis. Altogether, these results suggest that the sensitivity of TUS can be lower in children who are overweight or older.

In this study, the diagnostic rate of TUS seemed to be lower for DU compared to that for GU. However, there was no significant difference in diagnostic accuracy of TUS according to the location of the lesion when patients were divided into those who were sus- pected to have peptic ulcer and those who were not suspected. In addition, there was no statistically significant difference in the sensitivity of TUS in diagnosing PUD according to the localization of the lesion between patients under $30 \mathrm{~kg}$ and those over 30 $\mathrm{kg}$. Inflammatory changes of the duodenal wall are generally known to have a lower ultrasound diagnostic rate than changes in the gastric wall. The location of the duodenum and its close relationship with other organs make it easier to miss or misinterpret abnormalities. In previous studies, comparisons of topography showed that TUS detected bowel wall inflammatory changes preferentially in the terminal ileum and colon whereas such changes in the duodenum, jejunum, and rectum were frequently missed $[18,19]$. The reason for the difference between results of this study and those of other studies is presumably because of the small number of patients enrolled in the present study.

This study has several limitations. First, TUS was performed on an on-call request in patients with acute abdomen or GI bleeding. The diagnostic quality of TUS might have been compromised by the presence of bowel gas and fecal material. Fluid-aided sonography of the stomach and duodenum may increase the diagnostic sensitivity [20]. Future investigations could use an oral administration of an echoic, cellulose-based, gastric ultrasound contrast agent as it has recently been suggested to be a valuable initial screening tool for GU [21]. Second, TUS is highly operator-dependent. Its diagnostic accuracy is influenced by sonographer experience. Third, since this is a retrospective, single-center study with a small number of patients, a prospective study with a large number of patients is needed to clarify the diagnostic value of TUS in the diagnosis of PUD in children.

In conclusion, TUS investigation of the stomach and duodenum is an efficient diagnostic method. If an upper GI tract disease is suspected in children weighing less than $30 \mathrm{~kg}$, a thorough ultrasound examination will be helpful to avoid unnecessary endoscopic examination and allow the most appropriate subsequent diagnostic procedure to be chosen. 
However, ultrasonography cannot replace endoscopy, especially in patients who are overweight. Even if TUS is negative, endoscopy should be considered in patients with clinically suspected PUD.

\section{ACKNOWLEDGEMENTS}

This work was supported by a 2-year research grant of Pusan National University.

\section{REFERENCES}

1. Sung JJ, Kuipers EJ, El-Serag HB. Systematic review: the global incidence and prevalence of peptic ulcer disease. Aliment Pharmacol Ther 2009;29:938-46.

2. Lanas A, Chan FKL. Peptic ulcer disease. Lancet 2017;390:613-24.

3. Nord KS. Peptic ulcer disease in the pediatric population. Pediatr Clin North Am 1988;35:117-40.

4. Blanchard SS, Czinn SJ. Peptic Ulcer Disease in Children. In: Kliegman R, Stanton BMD, St Geme J, Schor NF, eds. Nelson Textbook of Pediatrics. 20th ed. Philadelphia: Elsevier, 2016:1816-9.

5. Hayden CK, Swischuk LE, Rytting JE. Gastric ulcer disease in infants: US findings. Radiology 1987;164: 131-4.

6. Herliczek TW, Raghavan D, McCarten K, Wallach M. Sonographic upper gastrointestinal series in the vomiting infant: how we do it. J Clin Imaging Sci 2011;1:19.

7. Gritzmann N, Hollerweger A, Macheiner P, Rettenbacher T. Transabdominal sonography of the gastrointestinal tract. Eur Radiol 2002;12:1748-61.

8. Lim JH, Jeong YM. Sonography of the stomach: an in vitro study to determine the anatomic cause of inner hyperechoic and hypoechoic layers of the gastric wall. AJR Am J Roentgenol 1994;162:335-8.

9. Lim JH, Lee DH, Ko YT. Sonographic detection of duodenal ulcer. J Ultrasound Med 1992;11:91-4.

10. Lorentzen T, Nolsøe CP, Khattar SC, Torp-Pedersen ST, Holm HH. Gastric and duodenal wall thickening on abdominal ultrasonography. Positive predictive value.
J Ultrasound Med 1993;12:633-7.

11. Swenson DW, Wallach M. Helicobacter pylori-associated antral gastritis and ulcer disease: imaging by computed tomography and ultrasound. Ultrasound Q 2012;28: 185-7.

12. Horton KM, Fishman EK. Current role of CT in imaging of the stomach. Radiographics 2003;23:75-87.

13. Sulowski C, Doria AS, Langer JC, Man C, Stephens D, Schuh S. Clinical outcomes in obese and normal-weight children undergoing ultrasound for suspected appendicitis. Acad Emerg Med 2011;18:167-73.

14. Hörmann M, Scharitzer M, Stadler A, Pokieser P, Puig $\mathrm{S}$, Helbich T. Ultrasound of the appendix in children: is the child too obese? Eur Radiol 2003;13:1428-31.

15. Josephson T, Styrud J, Eriksson S. Ultrasonography in acute appendicitis. Body mass index as selection factor for US examination. Acta Radiol 2000;41:486-8.

16. Yu SH, Kim CB, Park JW, Kim MS, Radosevich DM. Ultrasonography in the diagnosis of appendicitis: evaluation by meta-analysis. Korean J Radiol 2005;6:26777.

17. Kotagal M, Richards MK, Flum DR, Acierno SP, Weinsheimer RL, Goldin AB. Use and accuracy of diagnostic imaging in the evaluation of pediatric appendicitis. J Pediatr Surg 2015;50:642-6.

18. Parente F, Greco S, Molteni M, Cucino C, Maconi G, Sampietro GM, et al. Role of early ultrasound in detecting inflammatory intestinal disorders and identifying their anatomical location within the bowel. Aliment Pharmacol Ther 2003;18:1009-16.

19. Hollerbach S, Geissler A, Schiegl H, Kullmann F, Lock G, Schmidt J, et al. The accuracy of abdominal ultrasound in the assessment of bowel disorders. Scand J Gastroenterol 1998;33:1201-8.

20. Joharjy IA, Mustafa MA, Zaidi AJ. Fluid-aided sonography of the stomach and duodenum in the diagnosis of peptic ulcer disease in adult patients. J Ultrasound Med 1990;9:77-84.

21. Liu Z, Guo J, Wang S, Zhao Y, Liu Z, Li J, et al. Evaluation of transabdominal ultrasound with oral cellulose-based contrast agent in the detection and surveillance of gastric ulcer. Ultrasound Med Biol 2017;43:1364-71. 